\title{
Factores de riesgo de la ideación suicida en adolescentes de media vocacional de dos Instituciones Educativas de la localidad de Fontibón. Un análisis desde la Psicología Forense ${ }^{1}$
}

\author{
Pescador Varón; Leidy Katerine ${ }^{2}$; Jiménez-Jiménez, William Alejandro.
}

\begin{abstract}
Resumen | En esta investigación se analizó los factores de riesgo presentes en la ideación suicida de 124 adolescentes de media vocacional, con edades comprendidas entre 14 a 18 años, mediante

la aplicación del Inventario PANSI en dos Instituciones Educativas de la localidad de Fontibón. Se realizó un estudio cuantitativo de diseño no experimental de tipo transversal descriptivo correlacional. Entre los resultados que se obtuvieron se evidencia correlación entre las variables que se midieron, considerando que los puntajes más altos son las causas que llevan a que los adolescentes presenten conducta suicida. Para el análisis de resultados se utilizó el programa SPSS Windows 17, en el cual se muestra que los factores más comunes de conductas suicidas en estas instituciones son: la tristeza, la soledad, la depresión, la ideación suicida y la desesperanza, acompañado de factores protectores como:
\end{abstract}

\section{Editado por}

William Alejandro Jiménez-Jiménez

Universidad de Boyacá, Colombia.

\section{Revisado por}

Jaiver Humberto Urrego

Universidad Manuela Beltrán, Colombia.
${ }^{2}$ Leidy Katerine Pescador

Varón $[\bowtie]$

3 William Alejandro JiménezJiménez
Psicóloga egresada de la Fundación Universitaria los Libertadores. Tesista de la presente investigación.

Asesor del trabajo de grado. Psicólogo de la Universidad Católica de Colombia. Magíster en Seguridad y Defensa Nacionales. Docente tiempo completo de la facultad de Psicología de la Fundación Universitaria los Libertadores. Contacto: wajimenezj@libertadores.edu.co.

\footnotetext{
${ }^{1}$ Artículo de investigación producto del trabajo de grado presentado para optar por el título de psicóloga, con calificación meritoria. Fundación Universitaria los Libertadores.
} 
la autoconfianza, la esperanza, la alegría, la satisfacción con la vida y el autocontrol. Enfocado hacia el campo de la psicología forense, se evidencia la ausencia de material teórico y empírico que argumente las evidencias encontradas en este estudio.

Palabras claves | Tentativa de suicidio; delincuente juvenil; factores de conducta criminal; conducta delictiva; psicología criminal.

Abstract | This research presents the risk factors, which are presented in the suicidal ideation in 124 vocational media participants, aged between 14-18 years, by applying the PANSI inventory in two educational institutions of the Fontibón locality. It was carried out a quantitative study of non-experimental design of descriptive correlational conducted. Among the results gotten, it is evidenced correlation between the variables measured, whereas the highest scores are the causes that lead to teenagers present suicidal behavior. For the analysis of outcomes the SPSS Windows 17 program was used, which shows that the most common factors of suicidal behavior in these institutions are: sadness, loneliness, depression, suicidal ideation and hopelessness, along with factors protectors such as self-confidence, hope, joy, satisfaction with life and self-control. Focused on the field of forensic psychology, the lack of theoretical and empirical material asserting the evidence found in this study evidence.

Key words | Suicide attempt; juvenile delinquent; factors of criminal conduct; criminal conduct; criminal psychology.

\section{Introducción}

El suicidio se ha calificado como un problema socio-cultural que se manifiesta desde que el hombre es hombre. Los mayas creían que a través del suicidio se lograba llegar al cielo. En algunas culturas, como la Romana el suicidio se consideraba una forma positiva de acabar con la vida para buscar la liberación de sus males. Otras religiones lo rechazan y consideran que la persona suicida no tiene perdón de Dios (Villagómez, Balcázar \& Paz, 2005) 
A principios del siglo XX surgen dos grandes teorías del suicidio postuladas por Emilio Durkheim y Sigmund Freud (citados por Moron, 1992). Para Durkheim el suicidio no es frecuente en una sociedad integrada y está asociado al cambio de valores de la sociedad en particular. Por su parte, Freud se interesó en la conducta del inconsciente humano; planteando que el individuo suicida recibe influencia de su ira inconsciente, no expresada hacia una persona amada.

Por ello, el estudio del suicido siempre ha estado presente en las intenciones de ser abordado como un problema social; es un fenómeno mundial que se manifiesta en la vida cotidiana de algunas personas afectando su entorno habitual. Esta conducta ha tenido un impacto estadístico de corte ascendente en las últimas décadas sobre todo en los adolescentes, ya que en esta etapa se presentan cambios fisiológicos y psicológicos que llevan al individuo a convertirse en personas vulnerables. Algunos de los factores de riesgo que rodean la presencia de conductas negativas están asociados con el consumo de sustancias psicoactivas, la depresión, los cambios de personalidad, entre otras; que comprometen su integridad física y psicológica llevándolos a pensar que el suicidio es la única salida a sus problemas (Villalobos \& Galvis, 2009).

A raíz de lo anteriormente presentado, han sido varias las aproximaciones realizadas por diferentes autores para definir lo que significa suicidio. (Cortés, Aguilar, Medina, Toledo \& Echemendia, 2010), Ulloa (1994, citado en Cruz, Roa y Sepúlveda, 2005), Poldinger (1968, citado en Christensen 1995), Organización Mundial de la Salud (2000, citado en Soler \& Gascón, 2005), Bulbena (1991, citado por Cruz, Roa Sepúlveda, 2005). De igual forma, con relación a la clasificación del suicidio, como lo trabajado por Schneiderman (1996, citado en Villagómez; Balcázar y Paz, 2005), Soler y Gascón (2005), Goldney (1984, citado en Casullo 2005), y Durkheim (2008, citado en Palacio 2010).

Sin embargo, para los efectos de la presente investigación se tomó la idea que plantea la Organización Mundial de la Salud - OMS (2002), la cual menciona que el suicidio es una las principales causas de mortalidad en el mundo, sobre todo en la población juvenil. Las causas más comunes para que un individuo se quite la vida son la depresión, baja tolerancia a la frustración, falta de atención por parte de las familias, problemas sentimentales, rechazo en el contexto escolar, la violencia social que se presencia a diario, antecedentes familiares relacionados con suicidio, el abuso de sustancias, entre otros (Soler y Gascón, 2005). Así, el factor psicológico de la depresión resulta ser uno de los detonantes que desencadena la decisión de 
suicidio en los adolescentes, debido a que genera desmotivación sobre su futuro, aumentando la desesperanza y desarrollo de pensamientos negativos que pueden ocasionar comportamientos auto-agresivos (Jackson, 1989).

De igual forma, las rupturas sentimentales que no finalicen en buenos términos, el vínculo social son también causas que influyen significativamente en el desarrollo psicológico de la persona. Todas estas experiencias generan conductas depresivas con pensamientos suicidas, síntomas asociados al trastorno de ansiedad, los cuales llevan a que se generen este tipo de conductas (Villalobos \& Galvis, 2009).

Según el anuario estadístico de la Organización Mundial de la Salud (2002, citado en el Instituto Nacional de Medicina Legal y Ciencias Forenses, 2012) en países europeos como Dinamarca, Islandia, Irlanda \& Suiza, se suicidan un millón de personas al año aproximadamente. Los datos de países latinos no se alejan de esta cifra, pues, Estados Unidos, Canadá, Brasil, Cuba y Uruguay, reportaron índices muy altos de suicidio en el 2002.

Por otra parte, diversos son los autores que han buscado obtener evidencia empírica para entender con mayor precisión el fenómeno del suicidio (Barbosa, 2011; González et al., 2010; Pérez, Téllez \& Vélez, 2012; Pasturino, Vallarino \& Lima, 2004). La OMS (2002, citado en el Instituto Nacional de Medicina Legal y Ciencias Forenses, 2012) afirma que a nivel mundial aproximadamente 3.000 personas se suicidan a diario, es decir, una cada 30 segundos, y aproximadamente 60.000 presentan intentos suicidas, encontrando como causa principal, la depresión. En las últimas décadas ha aumentado un $60 \%$, especialmente, en países en desarrollo, seguido de los homicidios y los accidentes. El suicidio es una de las principales causas de muerte entre los 15 y los 34 años a nivel mundial. En los adolescentes se evidencia en un tercio de los países del mundo, razón que ha llevado a determinar que el grupo de mayor riesgo son los jóvenes.

De igual forma, con relación a las estadísticas del Instituto Nacional de Medicina Legal y Ciencias Forenses (2013) se presentaron entre los meses de enero y mayo 67 casos en niños, niñas y adolescentes de suicidio en Colombia. Entre ellos se encuentran 40 hombres y 27 mujeres, en un rango de edad de 15 y 17 años. El día en que más se presentaron los suicidios fueron los jueves, con 19 suicidios a las 6 de tarde y las 12 de la noche y el lugar más frecuente para cometer el acto suicida han sido sus residencias.

$\boldsymbol{\epsilon} \mid 106$ 
Respecto a Colombia el intento de suicidio ha aumentado a 1.2 \%. La población con mayor riesgo son adolescentes en un rango de edad entre 16 a 21 años. En los últimos años se aumentó en niños menores de 14 años (Gómez, et al., 2002).

Gráfica 1 / Suicidios en Colombia 2000-2010 según el Instituto Nacional de Medicina Legal y Ciencias Forenses (2010)

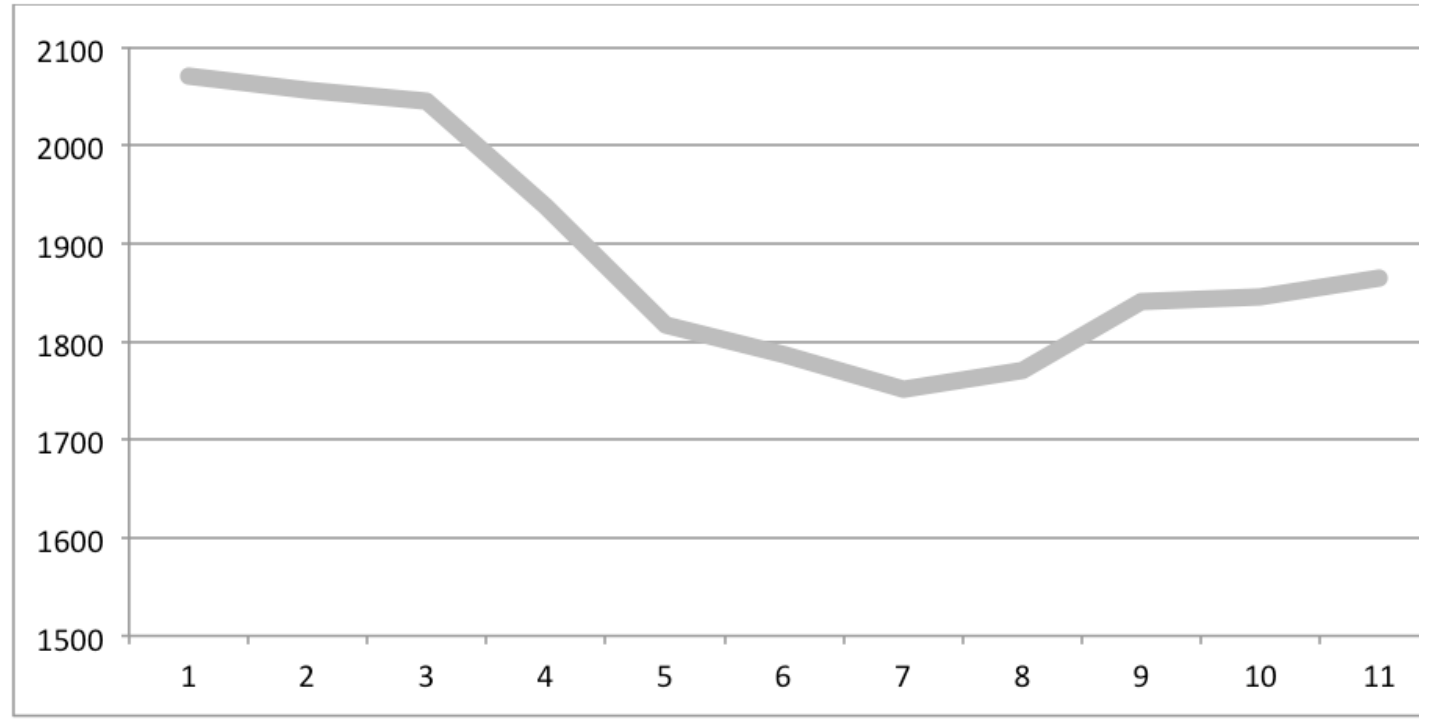

De acuerdo con las estadísticas arrojadas por el Instituto Nacional de Medicina Legal y Ciencias Forenses (2000, 2001, 2002, 2003, 2004, 2005, 2006, 2007, 2008, 2009, 2010, 2011 y 2012) el índice de suicidios ha tenido una curva muy representativa en el contexto colombiano. Según esta entidad los suicidios reportados fueron los siguientes: 2.070, 2.056, 2.045, 1.938, 1.817, $1.786,1.751,1.771,1.841,1.845,1.864,1.889$ y 1.901.

Las estadísticas de las conductas suicidas desde el año 2000 hasta la actualidad, muestran cómo el suicidio ha mantenido una traza de criminalidad estable, a pesar de los leves descensos en sus números, el comportamiento se mantiene muy similar en los años citados anteriormente. En los últimos años el fenómeno comienza a evidenciarse en el grupo de 15 a 24 años de edad 
de manera considerable. Realizando la comparación por género, los resultados muestran que por cada 4 hombres 1 mujer se suicida, esta información se mantiene desde el año 2000.

De igual forma, se evidencia que las regiones con mayor índice de suicidios son Antioquia, Bogotá y Valle del Cauca, con relación a los demás departamentos y ciudades de Colombia. Por otro lado, los factores más comunes que llevan a este tipo de actos son la falta de atención por parte de su familia, el rechazo en el colegio, la falta de trabajo, malas relaciones sentimentales, entre otras.

Por ello, el suicidio se ha convertido en una problemática social, ya que abarca diferentes aspectos del individuo, incluyendo cultura, emociones, religión, entre otras; llevando a que se genere una urgencia de salud pública a nivel mundial, que busca transformar el pensamiento de las nuevas generaciones y así evitar este tipo de conductas (Palacio, 2010). Debido a esta problemática, la OMS ha establecido el 10 de septiembre como el día mundial de prevención del suicidio, patrocinada por la Asociación Internacional para la Prevención del Suicidio. El propósito es generar compromisos y tomar medidas pertinentes para prevenir estas conductas en las personas que han intentado suicidarse, garantizándoles tratamientos y terapias efectivas que logren prevenir próximos pensamientos suicidas (Instituto Nacional de Medicina Legal y de Ciencias Forenses, 2012).

Por otro lado, la epidemiología ha aportado datos importantes para la investigación de la conducta suicida en los adolescentes, dado que se pueden identificar los factores de riesgo que se presentan en las personas con ideación suicida, diferenciándolas en variables sociodemográficas, psicopatológicas y sociales. Casullo (2007) identifica las siguientes variables socio-demográficas presentes en el suicidio: 1. El género, 2. Edad, 3. Estado civil, 4. La salud mental. La mayoría de los suicidios se han presentado por fuertes impulsos repentinos de acabar con la vida, en consecuencia a ideaciones suicidas que hayan estado presentes con anterioridad. La otra cara de la moneda, de acuerdo con lo afirmado por Villalobos y Galvis (2009) refieren a que los actos suicidas cuidadosamente elaborados son escasos.

Justamente con lo mencionado anteriormente, un factor de riesgo es cualquier característica que posee el individuo o comunidad directamente relacionada con una elevada probabilidad 
de dañar la salud, Alcantar (2002). Según Gómez et al. (2002) los factores de riesgo se dividen en factores modificables y factores inmodificables. Por un lado, los modificables están relacionados con los factores psicológicos, psicopatológicos, sociales, los cuales pueden transformarse clínicamente; mientras que, los inmodificables se relacionan con el propio sujeto o el grupo social al que pertenecen, se caracterizan porque perduran en el tiempo y su cambio es independiente al clínico. Entre factores de riesgo importantes se encuentran la existencia de trastornos mentales y haber tenido varias tentativas de suicidio previas.

Así, los factores de riesgo en el suicidio, son aquellos que determinan el grado de vulnerabilidad que presenta una persona para cometer el acto suicida, en ellos se incluyen situaciones contextuales, socioculturales y psicopatologías específicas, Alcantar( 2002).

Tabla 2 / Clasificación de factores de riesgo suicida modificable e inmodificable de Alcantar (2002)

MODIFICABLE

INMODIFICABLE

» Trastorno afectivo

» Esquizofrenia

» Trastorno de ansiedad

»Abuso de sustancias

» Trastorno de personalidad

» Otros trastornos mentales

»Salud física

» Dimensiones psicológicas
» Heredabilidad

»Sexo

» Edad:

- Adolescentes y adultos jóvenes

- Edad geriátrica

»Estado civil

» Situación laboral y económica

»Creencias religiosas

» Apoyo social

» Conducta suicida previa 
Paralelamente, Moya (2010) menciona diferentes factores de riesgo que se relacionan con la conducta suicida, los cuales son: 1. Factores individuales, 2. Factores psicológicos, 3. Factores genéticos y biológicos, 4. Enfermedades físicas o discapacidad, 5. Factores de riesgo familiares y contextuales, 6. Factores socio-familiares y ambientales, 7. Historia de maltrato físico o abuso sexual, 8. Orientación sexual, y 9. Acoso por parte de iguales.

Por otra parte, con relación a los factores precipitantes se entienden como ciertos eventos de la vida que tienen que ver con acontecimientos estresantes, factores psicológicos vitales o el fácil acceso, medios o métodos de suicidio, que sirven como elemento facilitador del suicidio (Moya, 2010). Entre los factores precipitantes más frecuentes que provocan la ideación suicida se presentan: la humillación, problemas interpersonales, el aislamiento social, trastornos mentales, distorsiones cognitivas y la vulnerabilidad (Villalobos \& Galvis, 2009). De esta manera, los factores protectores son los que disminuyen la tendencia al suicidio, en presencia de factores de riesgo. Según Moya (2010) existen dos tipos: 1. Factores internos (personales), y 2. Factores externos (sociales o medioambientales).

El fenómeno de la ideación suicida ha estado presente a lo largo de la historia de la humanidad, ha ido generando una preocupación en todos los ámbitos de las disciplinas científicas, debido a que se generan consecuencias en las dinámicas psicosociales de la persona, llevándola a que presente este tipo de conducta como única solución a sus problemas (Cruz, Roa y Sepúlveda, 2005). La mayoría de las personas que presentan estos comportamientos están abrumadas por situaciones complicadas y tienen dificultad para encontrar soluciones. El problema principal no es que quieran morir sino que no encuentran motivos para seguir viviendo (Casullo, 2007). Algunas investigaciones que soportan lo anteriormente dicho fueron las realizadas por Núñez y Olivera (2008), Cortés et al. (2010), Arias y Marcos (2008), Jiménez y Ruiz (2011), y Pérez, Téllez y Vélez (2012).

De esta forma, el intento suicida es un acto de autodestrucción con intención de ocasionar daños físicos y psicológicos en la persona. En este proceso se presentan pensamientos de toda índole para cumplir con el objetivo deseado (Stengel, 1965, citado en Garcia, 2006). De forma paralela, Kreitman (1969), citado en (Alcantar, 2002) afirmó que el intento de suicidio se identifica dentro de las conductas suicidas no fatales, es decir, los parasuicidios. El intento de 
suicidio es un acto no mortal, donde el individuo tiene la intensión de autolesionarse o ingerir medicamentos con dosis muy elevadas a las sugeridas por un profesional, teniendo como objetivo acabar con su vida.

Por otro lado, Clemente y González (1996) citados en Villalobos y Galvis (2009) mencionan que los intentos suicidas pueden ser entendidos desde tres puntos de vista: 1. Como síntoma de enfermedad mental, 2. Como estrés, 3. Como crisis. Marchiori (1998), citado en (García, 2006) menciona que la ideación suicida es un comportamiento que pone en riesgo la vida de la persona que lo ha intentado, presentándose de diferentes maneras y con diversas características, clasificándola en cuatro fases: 1 . Intento gravísimo de suicidio, 2. Intento grave, 3. Intento leve, y 4. Intento sin daño.

\section{Instrumento PANSI}

A partir de lo presentado anteriormente, donde se observa la importancia del estudio científico de la conducta suicida, teniendo en cuenta los factores precipitantes que lo conducen, Osman et al. (1998), diseñaron un cuestionario que contiene 14 ítems, el cual mide la frecuencia con que se presenta la ideación suicida en los adolescentes y adultos, incorporando pensamientos positivos y negativos de riesgo. Este inventario positivo y negativo de Ideación Suicida (PANSI), se divide en dos sub-escalas: positivos ideación (PANSI-PI; seis puntos) y la ideación suicida negativo (PANSI-NSI; ocho ítems). Cada elemento consta de cinco opciones de respuesta de auto-evaluación anotado en una escala de 0 (nunca) a 4 (siempre). Las puntuaciones totales en las sub-escalas puede ser calculado mediante la suma de todos los elementos. Las puntuaciones más altas representan ideación positiva respecto al suicidio y la ideación suicida negativa respectivamente. Los autores llegaron a la conclusión de que el PANSI es un instrumento válido y fiable para la detección y el riesgo de la ideación suicida, y qué factores están presentes en los jóvenes para tomar esta decisión.

De acuerdo con lo anterior, Villalobos y Galvis (2010) explicaron las dos sub-escalas que se evaIúan en el cuestionario PANSI: 1. Pensamientos negativos (Mide los pensamientos que hacen referencia a la conducta suicida, tales como el fracaso, la desesperanza, la baja auto eficacia, la frustración o la tristeza), y 2. Pensamientos positivos (En ellos se destaca la presencia de razones 
protectoras para no realizar un acto suicida, se presenta el sentido de control, la autoconfianza, las expectativas positivas, la alegría y la satisfacción con la vida). Así, Villalobos y Galvis (2010) mencionan que para realizar un estudio estadístico, es adecuado utilizar el Análisis Factorial Exploratorio (AFE), con el fin de evaluar la validez de la prueba. El AFE se usa para tratar de descubrir la estructura interna de un número relativamente grande de variables y factores asociados a estas; es el tipo de análisis factorial más común.

Durante años se han realizado diversos estudios cuyo objetivo ha sido demostrar la fiabilidad y validez de esta prueba en adolescentes con riesgo suicida de diferentes países, en donde se ha evidenciado que este cuestionario es confiable para su aplicación, debido a que muestra con qué frecuencia se presenta la ideación suicida, los pensamientos positivos y negativos que rodean al individuo para que tengan este tipo de comportamiento. Algunas de las investigaciones realizadas fueron las desarrolladas por Osman et al. (1998); Villalobos y Galvis (2010); Chang, Lin y Chou (2009).

A partir de lo anteriormente mencionado, en donde se visualiza la magnitud y la densidad del problema del suicidio en adolescentes, se propuso como objetivo general de investigación, identificar cuáles son los factores de riesgo presentes en la ideación suicida de adolescentes de media vocacional, con edades comprendidas entre 14 a 18 años, mediante la aplicación del Inventario PANSI en dos instituciones educativas de la localidad de Fontibón.

\section{Método}

\section{Diseño}

Para los propósitos de la presente investigación cuantitativa, se utilizó un diseño no experimental de tipo transversal descriptivo correlacional, ya que de acuerdo con lo planteado por Hernández, Fernández y Baptista (2010), es descriptivo cuando se busca especificar las propiedades y las características de las personas y al mismo tiempo correlacionar dado que busca conocer la relación que existe entre dos o más conceptos bajo un mismo contexto. 


\section{Paradigma epistemológico}

De acuerdo con lo planteado en el objetivo y en los lineamientos del problema de investigación del presente estudio, el paradigma que soporta el desarrollo y fundamentación es el Empírico- analítico, ya que este permite describir, explicar y predecir sucesos mediante mediciones formales no contradictorias (Inche et al., 2003).

\section{Muestra}

Para esta investigación, se utilizó un muestreo no probabilístico de tipo intencional o por conveniencia, porque de acuerdo con lo planteado por Casal y Mateu (2003), este tipo de muestreo se caracteriza por la ausencia de métodos aleatorios y en donde la muestra tiene características similares a la población objeto de estudio.

\section{Participantes}

Los participantes de la presente investigación fue un grupo de adolescentes $(n=124)$ de dos colegios Distritales de la localidad de Fontibón, de los grados décimo y undécimo con edades comprendidas entre 14 a 18 años.

\section{Instrumento}

Como único instrumento de evaluación se utilizó el Inventario de Ideación Suicida Positiva y Negativa (Positive and Negative Suicidal Ideation - PANSI). La aplicación de este instrumento se hizo en formato papel durante un período de 30 minutos aproximadamente en las aulas respectivas de los estudiantes.

\section{Procedimiento}

El procedimiento utilizado para la aplicación del inventario PANSI consistió en dos fases: una primera fase de acercamiento a las Instituciones Públicas y solicitud del permiso para la aplicación del inventario y una segunda fase la aplicación a los adolescentes. 
En la primera fase, el acercamiento se hizo a dos instituciones de educación secundaria ubicado en la localidad de Fontibón durante el mes de noviembre. Luego de haber hablado con las directivas y haber explicado los alcances de la investigación, se contó con la autorización para la aplicación del inventario PANSI a los estudiantes de decimo y undécimo grado.

En la segunda fase, se asistió a los colegios en el mes de enero para la aplicación del inventario PANSI a los estudiantes de décimo y undécimo grado, amparado en la ley 1090 de 2006 en su título II artículo 5 (sobre la confidencialidad) se les indicó en qué consistía la prueba, junto con el objetivo de la investigación y se obtuvo datos de un total de 124 estudiantes activos de ambas instituciones. La aplicación del inventario se realizó de manera individual y auto-aplicado en cada uno de los salones, en un promedio de 30 minutos.

\section{Resultados}

Partiendo de los supuestos adquiridos en esta investigación, en donde se buscó identificar cuáles fueron los factores de riesgo presentes en la ideación suicida de adolescentes de media vocacional, con edades comprendidas entre 14 a 18 años, mediante la aplicación del Inventario PANSI en dos instituciones educativas de la localidad de Fontibón, se llevó en una primera instancia para el desarrollo de los resultados, un análisis descriptivo de las variables de estudio y luego un análisis desde el SPSS versión 17.

Con relación a las características de la muestra estudiada, se evidencia que la muestra estuvo conformada por un total de 124 adolescentes, donde el rango de edad estaba entre los 14 y 18 años. De igual forma, en la tabla 3 se presenta las propiedades descriptivas del estudio de la variable edad con relación a la muestra estudiada. Se encontró que de los 124 adolescentes estudiados, el género femenino tuvo la mayor frecuencia (77 mujeres) en comparación del género masculino (47 hombres). Con relación a lo descriptivo de los géneros, estas propiedades están enfocadas a los valores de tendencia central como lo son: la media, la moda y la mediana, junto con los valores máximos y mínimos de las edades por género acompañado de los valores relacionados con la variación y desviación muestral. 
Tabla 3 / Características estadísticas de la muestra

\begin{tabular}{ccccccccc}
\hline \hline & N & MEDIA & MEDIANA & MODA & $\begin{array}{c}\text { V. } \\
\text { MÁXIMO }\end{array}$ & V. MÍNIMO & VARIANZA & $\begin{array}{c}\text { D. } \\
\text { ESTÁNDAR }\end{array}$ \\
\hline \hline Hombre & 47 & 16,22 & 16 & 16 & 18 & 14 & 0,92349479 & 0,97137571 \\
Mujer & 77 & 16,1472 & 16 & 16 & 18 & 15 & 0,78023275 & 0,88910009 \\
\hline
\end{tabular}

\section{Análisis Factorial Exploratorio del SPSS Windows 17}

Para el análisis de resultados se utilizó el Análisis Factorial Exploratorio del programa SPSS Windows versión 17, con el fin de obtener datos estadísticos de las variables utilizadas en la prueba aplicada en las Instituciones Educativas de la localidad de Fontibón.

De esta manera, en la tabla 4 se puede evidenciar que existe correlación entre las variables, debido a que se utilizó la prueba de Kaiser-Meyer-Olkin, en la cual se obtuvo un coeficiente de .829 que se acerca a 1 , indicando que existe asociación entre las variables utilizadas. Por otro lado, en la prueba de esfericidad de Bartlett se obtuvo como resultados ( $\chi 2: 702.18$; gl: 91).

Tabla 4 / Análisis estadístico de correlación de variables inventario PANSI

\begin{tabular}{ll}
\hline \hline \multicolumn{2}{c}{ CORRELACIÓN DE VARIABLES } \\
\hline \hline Medida de adecuación muestral de Kaiser-Meyer-Olkin. & .829 \\
Prueba de esfericidad de Bartlett Chi-cuadrado aproximado & 702.218 \\
$\mathrm{gl}$ & 91 \\
\hline
\end{tabular}

Los siguientes resultados se obtuvieron de una escala de 0 a 1 en donde se toma como valor principal el 1 , porque muestra la validez entre las variables que fueron utilizadas en el inventario de ideación suicida positiva y negativa; el PANSI, ya que los valores que más se acercan al 1 son los factores que utilizan los adolescentes para ejecutar o prevenir la conducta suicida. 
Por otro lado, en la tabla 5 se evidencia el análisis factorial de los ítems de ideación suicida negativa, donde prevalecen los pensamientos negativos que tienen los adolescentes de las instituciones educativas de la Localidad de Fontibón, frente a la conducta suicida, ya que la consideran como única salida a sus problemas. Entre los factores de riesgo con más puntuación se evidencia la tristeza en un rango de 962; la soledad y la depresión con puntuación de 961; entre las puntuaciones intermedias se refleja la ideación suicida con valores de 895 a 749, y por último se encuentra la desesperanza con .642.

Tabla 5 / Análisis factorial de ideación suicida negativa

\begin{tabular}{cc}
\hline \hline ÍTEMS & ANÁLISIS FACTORIAL \\
\hline \hline
\end{tabular}

4. ¿Te sentiste tan triste por tu relación con alguien importante, que quisiste estar muerto?

.962

11. ¿Te sentiste tan solo(a) o tan triste que querías matarte para así terminar con ese sufrimiento?

7. ¿Pensaste en matarte porque no encontraste una solución a un problema personal?

1. ¿Has considerado seriamente matarte porque no pudiste cumplir con lo que otras personas esperaban de ti?

9. ¿Pensaste en matarte porque viste que tu vida era un fracaso?

5. ¿Pensaste en matarte porque no pudiste hacer algo que era muy importante en tu vida?

10. ¿Pensaste que tus problemas eran tan graves que la única opción que tenías era suicidarte?

3. ¿Pensaste en matarte porque no tenías esperanza en el futuro?

De igual manera, en la tabla 6 se puede evidenciar el análisis factorial de la ideación suicida positiva, donde se reflejan los pensamientos de protección que utilizan los adolescentes de las instituciones educativas distritales de la Localidad de Fontibón, para prevenir la ideación suicida; entre las puntuaciones más altas se encuentra la autoconfianza con un valor de 1.101, la esperanza con un puntaje de 1.054, la alegría con un rango de 1.048; en las puntuaciones

ह 116 
intermedias se evidencia la satisfacción con la vida con un 0.954 y en la calificación baja se presenta el autocontrol con 0.821 a 0.819.

Tabla 6 / Análisis factorial de ideación suicida positiva

\begin{tabular}{lc}
\hline \hline \multicolumn{1}{c}{ ÍTEMS } & ANÁLISIS FACTORIAL \\
\hline \hline $\begin{array}{l}\text { 12. ¿Tuviste confianza en las capacidades que tenías para enfrentar la } \\
\text { mayoría de problemas de tu vida? }\end{array}$ & 1.101 \\
$\begin{array}{l}\text { 6. ¿Tuviste esperanza en el futuro porque las cosas estaban saliendo como } \\
\text { tú querías? }\end{array}$ & 1.054 \\
$\begin{array}{l}\text { 8. ¿Te sentiste alegre porque te estaba yendo bien en el colegio o en el } \\
\text { trabajo? }\end{array}$ & 1.048 \\
$\begin{array}{l}\text { 13. ¿Sentiste que valía la pena vivir la vida? } \\
\text { 14. ¿Tuviste confianza en lograr tus metas en el futuro? }\end{array}$ & .954 \\
$\begin{array}{l}\text { 2. ¿'Has sentido que tenías el control de la mayoría de las situaciones de tu } \\
\text { vida? }\end{array}$ & .821 \\
\hline
\end{tabular}

\section{Discusión}

Se encontró que dentro de los factores de riesgo presentes en las conductas suicidas en los adolescentes de las dos instituciones educativas de la Localidad de Fontibón se manifiesta la desesperanza, la soledad, la tristeza y la pérdida de control como las causas que más intervinieron para que la persona tomara este tipo de conductas buscando solución a sus problemas. Por otro lado, se obtiene los factores protectores que son utilizados para prevenir la ideación suicida, predominando el autocontrol, la alegría y la satisfacción con la vida.

Paralelamente, para la investigación de los resultados se utilizó el programa SPSS Windows 17, con el objetivo de obtener el análisis factorial exploratorio de la prueba, donde se evidenció que existe correlación entre las variables que se midieron, alcanzando un puntaje de 0.829 , el cual se logra con la medición de una escala de 0 a 1 en la cual se toma como valor principal el 1. 
De esta manera, se confirmó la validez que tuvo la prueba aplicada en las dos instituciones educativas de la Localidad de Fontibón, mostrando que los valores con puntajes más altos son las causas que llevan a que los adolescentes presenten conducta suicida.

Por otro lado, Villalobos y Galvis (2010), muestran que los factores protectores que previenen la ejecución de la conducta suicida en los adolescentes tienen puntuación alta en la prueba. Entre los más comunes se encuentran: el sentido de control, la autoconfianza, las expectativas positivas, la alegría y la satisfacción. Comparado con los resultados de esta investigación se evidencia que la autoconfianza con un valor de 1.101, la esperanza con 1.054, la alegría con un rango de 1.048, son las conductas que más utilizan los adolescentes de las dos instituciones educativas distritales de la Localidad de Fontibón para prevenir la ideación suicida, seguido por la satisfacción con la vida con un 0.954 y el autocontrol con 0.821 a 0.819 .

Con respecto a lo anterior, se puede mencionar que existen diversos factores de riesgo que rodean la conducta suicida; entre los más importantes se encuentran: los vínculos familiares, sociales y emocionales, debido a que tienen gran influencia para que se tome este tipo de decisión. Se pudo evidenciar también que la población más vulnerable para cometer este tipo de conducta son los adolescentes, ya que se encuentran más propensos a problemas que los rodean a diario y no buscan salidas adecuadas para solucionarlos.

Finalmente, es necesario tener en cuenta los factores protectores que se pueden utilizar para prevenir la conducta suicida, ya que así se puede disminuir esta problemática que se encuentra a nivel mundial y se le da más valor a la vida.

Ahora bien, con relación al componente forense, muestra que en los estudios desarrollados anteriormente junto con los obtenidos en esta investigación, hay consenso en torno a los factores de riesgo y de protección que tienen los adolescentes en torno a la ideación suicida. Sin embargo, los aportes desde la psicología jurídica como campo de aplicación de la psicología como ciencia son poco visibles. De acuerdo con lo planteado por Sierra, Jiménez y Buela-Casal (2006), los alcances que tiene la psicología forense en su práctica profesional está, entre otros, el de:

$\boldsymbol{\epsilon} \mid 118$ 
Establecer unas pautas más idóneas para evaluar la conducta humana dentro del ámbito judicial (teniendo en cuenta los distintos factores que afectan dicha conducta, como por ejemplo la salud mental, motivación, consecuencias, contexto social, responsabilidad, seguridad, antecedentes culturales, etc., (pág. 74).

Así, desde la psicología forense lo que se busca es aportar la mayor cantidad de evidencias posibles que sean de utilidad para el sistema de justicia. En este orden de ideas, Urra (2002), citado por (Jiménez, 2008) expone que la actuación del psicólogo forense se encuentra establecida dentro de los siguientes lineamientos: exploración, evaluación, diagnóstico de las relaciones, pautas de interacción, personalidad, inteligencia, aptitudes, actitudes, entre otras características de la persona implicada en un proceso judicial.

Posiblemente la técnica que más se acerca al abordaje del suicido desde el punto de vista forense es la autopsia psicológica, la cual cumple un papel fundamental en el ámbito jurídico, debido a esto se ha ido vinculando como un procedimiento importante que aclara muertes dudosas. Gómez y Sanz (2000), citado en (Andrade, Téllez y Giraldo, 2013) definen la autopsia psicológica como un método de análisis, que puede dar respuesta a los motivos psicosociales que llevan a la muerte por suicidio. No obstante, en Colombia, la falta de autopsias psicológicas frecuentes, influye para que no se puedan detectar las variables asociadas al suicidio, encontrándose sin datos porcentuales en los registros de Medicina legal Forenses.

En este orden de ideas, lo que se busca concientizar desde la psicología forense hacia la problemática del comportamiento del suicidio y su manifestación de los factores de riesgo y protectores que conducen a la ideación, es en torno a dos factores propios de la evaluación psicológica forense; por un lado la peligrosidad, y por el otro la reincidencia (en caso que el acto suicida sea fallido).

Por ello, al hablar de peligrosidad, la cual es entendida como la tendencia de cometer actos peligrosos, manifestada en conductas que sean dañinas para sí mismos o para otros, durante un período de tiempo determinado (Jiménez, 2008). De igual forma, con relación al factor de reincidencia, surge como variables de análisis un listado de factores que están presentes en la comisión de un hecho delictivo. Por ejemplo, Grath (1991) afirma que estas son algunas de las 
variables que conducen a la reincidencia: 1 . No tener un empleo; 2 . Pertenecer a clase socioeconómica baja; 3 . Inexistencia de los lazos familiares; 4 . La presencia de parafilias; 5 . Historial penal; 6. Uso de la fuerza para cometer los actos delictivos y 7. No estar casado.

De igual forma, coincide De Castro y Ponce (2009), citados por (Amar y Tirado 2011), al afirmar que la psicología forense está dedicada al peritaje, en busca de responder a las preguntas de la justicia encaminada hacia los siguientes objetivos: a la inimputabilidad, a la perturbación y capacidad psicológica de los actores jurídicos, a la veracidad del testimonio, a la peligrosidad y a la reincidencia, al análisis de los factores de atenuación o agravación punitiva.

En este sentido, el hablar de la problemática de la ideación suicida dentro del contexto forense, y teniendo como base los datos obtenidos en la población adolescente objeto de estudio de la investigación, resulta inequívoco pensar que efectivamente hacen falta tomar medidas que permitan minimizar el riesgo desde lo forense (es decir desde la evaluación y su intervención legal) para aportar datos desde la evidencia al Sistema Jurídico Colombiano y determinar las repercusiones de este tipo de conductas en el contexto socio-jurídico.

Finalmente, resulta interesante abordar la ideación suicida desde la psicología forense porque permite comprender la dinámica de este tipo de conducta, y teniendo como base un sustento clínico, fundamentar el actuar profesional del psicólogo forense, no solo en mirar hacia la evaluación de las conductas manifiestas, sino de la minimización de las mismas a partir de la predicción.

\section{Referencias}

Alcantar, M.I. (2002). Prevalencia del intento suicida en estudiantes adolescentes y su relación con el consumo de drogas, la autoestima, la ideación suicida y el ambiente familiar. México. (Tesis de pregrado - Facultad de psicología -Universidad Autónoma de Mexico). Recuperado de: http://www.uade.inpsiquiatria. edu.mx/pagina_contenidos/tesis/tesis_isabel_alcantar.pdf

Amar, J. \& Tirado, D. (2011). Definiendo la Psicología Forense. En: Psicologia Forense: Estudio de la mente criminal. Edith Aristizábal y José Amar (Compiladores). Colombia: Ediciones Uninorte.

$\boldsymbol{\epsilon} \mid 120$ 
Andrade, J., Téllez, C y Giraldo, L. (2013). La Autopsia Psicológica en Colombia: Exploración Psicobiográfica del Suicidio. Revista Internacional de Psicología, 12(2), 13-24. Recuperado de: http://www.revistapsicologia. org/index.php/revista/article/view/70/67

Arias, M.; et al. (2008). Modificación de conocimientos sobre conducta suicida en adolescentes y adultos jóvenes con riesgo. MEDISAN, 13(1), 3-5. Recuperado de: http://scielo.sld.cu/scielo.php?script=sci_arttext\&p id=S1029-30192009000100005

Barbosa, L. (2011). Suicidio: un reto para las comunidades educativas. Bogotá. Pontificia Universidad Javeriana. Recuperado de: http://scielo.org.co/scielo.php?pid=S0120-05342012000200016

Casal, J. y Mateu, E. (2003). Tipos de muestreo. Revista epidemiológica de medicina preventiva,1, 3-7. Recuperado de http://minnie.uab.es/ veteri/21216/TiposMuestreo1.pdf

Casullo, M. (2007). Ideaciones y comportamientos suicidas en adolescentes: una urgencia social. Recuperado de: http://www.scielo.org.ar/scielo.php?pid=S1851-16 862005000100017\&script=sci_arttext

Chang, H., Lin, C. y Chou, K. (2009). Chinese Version of the Positive and Negative Suicide Ideation: Instrument Development. Journal of Advanced Nursing 65(7), 1485-1496. Recuperado de: http://www.researchgate.net/publication/24441239_Chinese_version_of_the_positive_and_negative_suicide_ideation_ instrument_development/file/72e7e516ff1b399e80.pdf

Christensen, C. (1995). Intencionalidad suicida. Trabajo de tesis. Universidad del Aconcagua, Facultad de Psicología.

Cortés, A., et al. (2010). Causas y factores asociados con el intento de suicidio en adolescentes. Epidemiología y Microbiología, 8 (1). PP. 15-23. Recuperado de: http://scielo.sld.cu/scielo.php?pid=S1561-300320100 00100003\&script=sci_arttext

Cruz, F., Roa, V. y Sepúlveda, G. (2005). Intento Suicida en Niños y Adolescentes. Revista Gaceta médica espirituana, 9, 23 - 35. Recuperado de: http://www.tesis.uchile.cl/tesis/uchile/2005/cruz_f/sources/cruz_f.pdf

González et al., (2010). Suicidio y género Antioquia (Colombia): estudio de autopsia psicológica. Revista colombiana de psiquiatría, 29(2), 252-267. Recuperado de: http://www.buscandoanimo.org/Descargas/Biblio/Autopsia/08_Suicidio\%20y\%20g\%E9nero\%20en\%20Antioquia\%20-\%20Gonzalez.pdf 
García, N. (2006). Ideación e Intento Suicida en estudiantes adolescentes y su relación con el consumo de drogas. (tesis de pregrado - Universidad Nacional Autónoma de México). Recuperado de: http://www.uade. inpsiquiatria.edu.mx/tesis/tesis_nayeli.pdf

Gómez et al., (2002). Factores asociados al intento de suicidio en la población colombiana. Revista Colombiana de Psiquiatría, XXXI (4), 283-298. Recuperado de: http://www.scielo.org.co/pdf/rcp/v31n4/v31n4a02.pdf

Hernández, R., Fernández, C. y Baptista, P. (2010). Metodología de la investigación. Quinta edición. México: Mc Graw-Hill.

Inche et al., (2003). Paradigma cuantitativo: un enfoque empírico- analítico. Universidad Nacional Mayor de San Marcos. Industrial data, 6(1), 23-37. Recuperado de: http://www.redalyc.org/articulo.oa?id=81606104

Instituto Nacional de Medicina Legal y Ciencias Forenses. (2000). Los suicidios. Forensis. Recuperado de: http:// www.medicinalegal.gov.co/images/stories/root/FORENSIS/2000/Suicidios.pdf

Instituto Nacional de Medicina Legal y Ciencias Forenses. (2001). Suicidios en Colombia. Forensis. Recuperado de: http://www.medicinalegal.gov.co/images/stories/root/FORENSIS/2001/Suicidios.pdf

Instituto Nacional de Medicina Legal y Ciencias Forenses. (2002). Los Suicidios en Colombia entre el libre albedrío y la prevención. Forensis. Recuperado de: http://www.medicinalegal.gov.co/images/stories/root/ FORENSIS/2002/Suicidios.pdf

Instituto Nacional de Medicina Legal y Ciencias Forenses. (2003). Conducta Suicida según ciclo vital. Forensis. Recuperado de: http://www.medicinalegal.gov.co/images/stories/root/FORENSIS/2003a/Suicidio.pdf

Instituto Nacional de Medicina Legal y Ciencias Forenses. (2004). Suicidio. Forensis. Recuperado de: http://www. medicinalegal.gov.co/images/stories/root/FORENSIS/2004/Suicidio.pdf

Instituto Nacional de Medicina Legal y Ciencias Forenses. (2005). Filicidio-suicidio. Un reto por estudiar. Forensis. Recuperado de: http://www.medicinalegal.gov.co/images/stories/root/FORENSIS/2005/Suicidio.pdf

Instituto Nacional de Medicina Legal y Ciencias Forenses. (2006). El suicidio y su prevención. Forensis. Recuperado de: http://www.medicinalegal.gov.co/images/stories/root/FORENSIS/2006/Suicidios.pdf 
Instituto Nacional de Medicina Legal y Ciencias Forenses. (2007). Epidemiologia de los suicidios en Colombia. Forensis. Recuperado de: http://www.medicinalegal.gov.co/images/stories/root/FORENSIS/2007/Suicidio.pdf

Instituto Nacional de Medicina Legal y Ciencias Forenses. (2008). Suicidio en Colombia. Forensis. Recuperado de: http://www.medicinalegal.gov.co/images/stories/root/FORENSIS/2008/Suicidio.pdf

Instituto Nacional de Medicina Legal y Ciencias Forenses. (2009). Suicidio en Colombia, Epidemiologia. Forensis. Recuperado de: http://www.medicinalegal.gov.co/images/stories/root/FORENSIS/2009/Suicidio.pdf

Instituto Nacional de Medicina Legal y Ciencias Forenses. (2010). La respuesta de muchos, la experiencia de la vida: el suicidio. Forensis. Recuperado de: http://www.medicinalegal.gov.co/images/stories/root/FORENSIS/2010/SUICIDIO.pdf

Instituto Nacional de Medicina Legal y Ciencias Forenses. (2011). Comportamiento del Suicidio en Colombia. Forensis. Recuperado de: http://www.medicinalegal.gov.co/images/stories/root/FORENSIS/2011/6-F-11-Suicidio.pdf

Instituto Nacional de Medicina Legal y Ciencias Forenses. (2012). Violencia autoinfligida desde el sistema médico-legal colombiano. Forensis. Recuperado de: http://www.medicinalegal.gov.co/images/stories/ root/FORENSIS/2012/5\%20suicidio\%20forensis\%202012.pdf

Instituto Nacional de Medicina Legal y Ciencias Forenses. (2013). Suicidio. Recuperado de: www.medicinalegal. gov.co/2013

Jackson, S. (1989). Historia de la melancolía y la depresión. Madrid: Ed. Turner.

Jiménez, M. y Ruiz, C. (2011). Desórdenes afectivos, crisis de identidad e ideación suicida en adolescentes. International Journal of Psychology and Psychological Therapy, 11(1), 33-56. Recuperado de: http://www. ijpsy.com/volumen11/num1/281/desordenes-afectivos-crisis-de-identidad-ES.pdf

Jiménez, W. (2008). Evaluación Psicológica Forense en la Seguridad Ciudadana. Psicología Colombiana - Sociedad Colombiana de Psicología, 25, 12 -16.

Moron, P. (1992). El suicidio. México. Lito arte S.A. Recuperado de: http://books.google.com.co/books?hl=es\&lr=\&i $d=Y k X p H v T 9 G X c C \& o i=f n d \& p g=P A 3 \& d q=M o r o n,+P .+(1992) .+E l+$ suicidio\&ots=33QeOv3eF\&sig=z5_ZK 1dBwLQRmCDqaGPkfsQlieY\#v=onepage\&q=Moron\%2C\%20P.\%20(1992).\%20El\%20suicidio\&f=false 
Moya, C. (2010). Guía de Práctica Clínica de Prevención y Tratamiento de la Conducta Suicida. Plan de Calidad para el Sistema Nacional de Salud del Ministerio de Sanidad, Política Social e Igualdad. Recuperado de: http://www.guiasalud.es/GPC/GPC_481_Conducta_Suicida_Avaliat_vol1_compl.pdf

Núñez, N. y Olivera, S. (2008). Perfil multidimensional de personas que han realizado intento de suicidio. Pensamiento psicológico, 4(10), 85-100. Recuperado de: http://www.redalyc.org/articulo.oa?id=80111670005

Osman et al., (1998). The Positive and Negative Suicide Ideation (PANSI) Inventory: development and validation. Psychological Reports, 61 (4), 783-793. Recuperado de: http://www.researchgate.net/publication/10965614_The_Positive_and_Negative_Suicide_Ideation_(PANSI)_inventory_psychometric_ evaluation_with_adolescent_psychiatric_inpatient_samples

Organización Mundial de la Salud. (2002). Informe mundial sobre la violencia y la salud: resumen. Organización Panamericana de la Salud. Recuperado de: http://www.who.int/violence_injury_prevention/violence/world_report/es/summary_es.pdf

Palacio, A. (2010). La Comprensión Clásica del Suicidio. De Emilse Durkheim a nuestros días. Revista Affectivo Societatis, 7 (12). Recuperado de: http://aprendeenlinea.udea.edu.co/revistas/index.php/affectiosocietatis/article/viewFile/6318/6520

Pasturino, B., Vallarino, V. y Lima, M. (2004). Estudio sobre suicidios consumados. Población usuaria del Hospital Viladerbo. Revista psiquiátrica, 68(2), 147-161. Recuperado de: http://www.spu.org.uy/revista/ dic2004/03_estudio.pdf

Pérez, I., Téllez, D. y Vélez. A. (2012). Caracterización de factores asociados con comportamiento suicida en adolescentes estudiantes de octavo grado, en tres colegios bogotanos. Revista Colombiana de Psiquiatría, 41(1), 26-47. Recuperado de: http://www.redalyc.org/pdf/806/80624093004.pdf

Sánchez, M. \& Dubroca, I. (2001). Evaluación psicológica Forense. Fuentes de información, abusos sexuales, testimonio, peligrosidad y reincidencia. Volumen 1. España: Amarú ediciones.

Sierra, J., Jiménez, E. y Buela, G. (2006). Psicología Forense: Manual de técnicas y aplicaciones. España: Biblioteca nueva.

\section{E $\| 124$}


Soler, P. y Gascón, J. (2005). Recomendaciones terapéuticas en los trastornos mentales. 3. Ed. España. Ed. Ars Médica. Recuperado de: http://www.suicidioprevencion.com/pdf/RTM3.pdf

Villagómez, R., Balcázar, A. y Paz, R. (2005). Suicidio en jóvenes. Revista de la Facultad de Medicina Universidad Nacional Autónoma de México, 2, 54 -55. Recuperado de: http://www.ejournal.unam.mx/rfm/no482/RFM48204.pdf

Villalobos y Galvis, F. (2009). Ideación suicida en jóvenes: formulación y validación del modelo integrador explicativo en estudiantes de educación secundaria y superior (Tesis doctoral, Universidad de Granada, España). Recuperada de http://hera.ugr.es/tesisugr/1805982x.pdf.

Villalobos y Galvis, F. (2010). Validez y fiabilidad del Inventario de Ideación Suicida Positiva y Negativa ? PANSI, en estudiantes colombianos. Universitas Psychologica, 9(2), 509-520. Recuperado de: http://www.scielo. org.co/pdf/rups/v9n2/v9n2a17

\section{Citación:}

Pescador Varón; L.; \& Jiménez-Jiménez, W. A. (2015). Factores de riesgo de la ideación suicida en adolescentes de media vocacional de dos Instituciones Educativas de la localidad de Fontibón. Un análisis desde la Psicología Forense. Enfoques, 1(2)

Recibido: 25 / 02 / 2015 |Aceptado: 31 / 03 / 2015. 